\title{
Endothelial injury and acquired aspirin resistance as promoters of regional thrombin formation and early vein graft failure after coronary artery bypass grafting
}

Robert S. Poston, MD, ${ }^{a}$ Junyan Gu, MD, PhD, ${ }^{a}$ James M. Brown, MD, ${ }^{a}$ James S. Gammie, MD, ${ }^{a}$ Charles White, MD, ${ }^{b}$ Lei Nie, PhD, ${ }^{c}$ Richard N. Pierson, III, MD, ${ }^{a}$ and Bartley P. Griffith, MD

From the Departments of Surgery, ${ }^{\text {a }}$ Radiology, ${ }^{\mathrm{b}}$ and Epidemiology, ${ }^{\mathrm{c}}$ University of Maryland School of Medicine and Baltimore VAMC, Baltimore, Md

R.S.P. is supported by a Scientist Development Grant from the American Heart Association $(0435318 \mathrm{~N})$ and by grants from Bayer Pharmaceutical Corp and the Office of Naval Research. Supplies were donated for the TEG (Haemoscope Corporation) and whole-blood aggregometry (Chronolog, Inc).

Read at the Eighty-fifth Annual Meeting of The American Association for Thoracic Surgery, San Francisco, Calif, April 10-13, 2005 .

Received for publication April 18, 2005; revisions received July 1, 2005; accepted for publication Aug 8, 2005.

Address for reprints: Robert S. Poston, MD, Assistant Professor of Surgery, Division of Cardiac Surgery, N4W94 22 S Greene St, Baltimore, MD 21201 (E-mail: rposton@ smail.umaryland.edu).

J Thorac Cardiovasc Surg 2006;131:122-30

$0022-5223 / \$ 32.00$

Copyright ( $\odot 2006$ by The American Association for Thoracic Surgery

doi:10.1016/j.jtcvs.2005.08.058
Objective: The predominant mechanism of early graft failure after coronary artery bypass grafting remains in doubt. Aspirin administered in the initial hours after coronary artery bypass grafting improves graft patency, implicating prostanoid synthesis in the pathogenesis. We hypothesized that synergy between endothelial disruption in the venous conduit and aspirin resistance would cause vein graft failure.

Methods: Aspirin resistance, defined by diagnostic findings on at least two of three separate assays, was serially assessed in 225 patients undergoing off-pump coronary artery bypass grafting. Endothelial cell integrity was determined in surplus segments obtained from 408 vein grafts. The deposition of intraluminal thrombin within the vein was determined by comparing serum F1.2 levels between the coronary sinus and the aorta after grafting. Intraoperative blood flow in the grafts was measured with transit-time technology, and patency was assessed with electrocardiographically gated multichannel computed tomographic coronary angiography on day 5 . Aspirin was the sole antithrombotic agent used during the study.

Results: Thrombosed grafts (16/408) showed more endothelial cell loss at the time of grafting than did those grafts that remained patent $(10.8 \% \pm 21.5 \%$ vs $51.4 \% \pm$ $39.1 \%$ integrity, $P<.01)$. Aspirin resistance occurred in 67 patients $(30 \%)$. Graft thrombosis was associated with aspirin resistance $(P<.04)$ and reduced endothelial integrity $(P<.01)$. These factors coexisted in 14 of 16 grafts that failed and were associated with elevated coronary sinus F1.2 levels.

Conclusion: Aspirin resistance and relatively compromised venous endothelial cell integrity together marked patients whose vein grafts failed within days after offpump coronary artery bypass grafting. These observations form a basis for identifying patients at risk and developing approaches to prevent vein injury or to selectively intervene in high-risk circumstances.

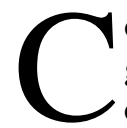
onditions that create nonlaminar or sluggish flow within the saphenous vein graft (SVG)—such as a large size mismatch between the conduit and coronary target, ${ }^{1}$ intrinsic disease in the outflow bed, or an abnormal anastomotic angle ${ }^{3}$ - influence SVG thrombosis after coronary artery bypass grafting (CABG). The most important cause of early attrition is thought to be inaccurate microvascular technique, particularly for grafts performed during off-pump CABG $(\mathrm{OPCAB}){ }^{4}$ However, randomized trials have shown no consistent influence of OPCAB on SVG thrombosis. ${ }^{5-7}$ In fact, we have found that SVG grafts still fail despite careful intraoperative screening for problems in technique with transit-time flow measurements. ${ }^{8}$ These observations implicate alternative features in the pathogenesis of graft thrombosis, such as conduit quality and systemic thrombogenicity. 


$$
\begin{aligned}
& \text { Abbreviations and Acronyms } \\
& \begin{array}{ll}
\text { CABG } & =\text { coronary artery bypass grafting } \\
\text { CS } & =\text { coronary sinus } \\
\text { CT } & =\text { computed tomography } \\
\text { EC } & =\text { endothelial cell } \\
\text { MA } & =\text { maximum amplitude } \\
\text { OPCAB } & =\text { off-pump coronary artery bypass grafting } \\
\text { PFA-100 } & =\text { platelet function analyzer } \\
\text { SVG } & =\text { saphenous vein graft } \\
\text { TEG } & =\text { thromboelastography } \\
\text { WBA } & =\text { whole-blood aggregometry }
\end{array}
\end{aligned}
$$

Endothelial cell (EC) integrity is known to be frequently impaired in veins used for bypass, ${ }^{9}$ and the proportion of denuded surface area correlates directly with reduced graft patency. ${ }^{10}$ Aspirin, a well known therapy for preventing graft failure, ${ }^{11}$ insufficiently inhibits platelets in many patients after cardiac surgery. ${ }^{12}$ These risk factors have rarely been evaluated in a systematic way after CABG and never for how they influence SVG patency in aggregate.

SVG thrombosis, variously reported at between $5 \%{ }^{6}$ and $40 \%{ }^{7}$ at 1 year, accounts for a substantial proportion of the well-known limitations of long-term patency associated with this conduit. Because of this demographically important opportunity for practice improvement, we initiated a prospective investigation of the mechanism of SVG thrombosis in patients undergoing OPCAB at a single institution. Preliminary analysis of this data set revealed that aspirin resistance ${ }^{13}$ and EC disruption ${ }^{10}$ were each associated with an increased risk of acute SVG failure. The hypothesis of this more current analysis of 250 unselected patients undergoing OPCAB was that these two risk factors interact to promote rapid thrombin formation within the SVG, synergistically increasing the risk of early thrombosis.

\section{Patients and Methods}

\section{Patient Enrollment and Data Management}

After institutional review board approval was obtained, all subjects provided informed consent before enrollment. From November 2002 until December 2004, a total of 410 patients were screened, and 133 patients excluded from the study for creatinine level 2.0 $\mathrm{mg} / \mathrm{dL}(\mathrm{n}=43)$, requirement for cardiopulmonary bypass $(\mathrm{n}=$ $37)$, refusal of consent $(n=32)$, and inability to obtain valid consent because of patient condition or emergency nature of surgery $(n=21)$. After enrollment, computed tomographic (CT) angiography was not performed in 52 patients because of heart rate greater than 100 beats/min or creatinine level greater than 2.0 $\mathrm{mg} / \mathrm{dL}(\mathrm{n}=28)$, patient withdrawal of consent $(\mathrm{n}=12)$, unavailability for follow-up $(\mathrm{n}=10)$, and patient death without an autopsy to confirm bypass graft patency $(n=2)$.

Demographic data, preoperative risk factors and medications, and intraoperative and postoperative data were prospectively recorded onto Teleform case report forms (TELEform Elite; Cardiff
Software Ltd, Vista, Calif), electronically scanned, and imported into a relational database.

\section{Surgical Technique}

Four surgeons (R.P., J.B., J.G. and B.G.), experienced in OPCAB, enrolled patients. After median sternotomy, the left internal thoracic artery was used in all patients; the saphenous vein was harvested with an endoscopic ( $\mathrm{n}=363$ venous conduits; VasoView5; Guidant Systems, Inc, Minneapolis, Minn) or open ( $\mathrm{n}=45$ venous conduits) approach, according to anatomic considerations. Conduits were flushed with heparinized saline solution after harvest, with no methods used to control the distending pressure. The anastomoses were performed first proximally with a partial occluding aortic clamp and then distally with suction-based exposure and stabilizing devices (Octopus 4.3; Medtronic, Inc, Minneapolis, Minn). Because of this protocol, little variation was present in the length of time from when each SVG was initially placed in solution saline storage until the proximal anastomosis was completed and the graft was reperfused with oxygenated blood (i.e. ischemic times similar). It was reperfused with oxygenated blood (proximal anastomosis completed). Heparin was given initially and every 30 minutes at a dose calculated to obtain an activated clotting time longer than 300 seconds and a heparin level of 2 $\mathrm{IU} / \mathrm{mL}$ according to protamine titration (HMS heparin assay cartridges; Medtronic). Heparin was reversed by half the dose of protamine calculated by heparin-protamine titration. Preoperative aspirin ( $325 \mathrm{mg} / \mathrm{d}$ orally) was continued and given within 6 hours of surgery as the sole antithrombotic agent used in the study.

\section{Intraoperative Blood Flow Analysis}

Blood flow and flow waveform were measured in each graft with transit time ultrasonography (Transonic Systems, Inc, Ithaca, NY). Waveforms were analyzed for pulsatility index ([maximum blood flow - minimum blood flow]/mean blood flow) and percentage diastolic flow with data acquisition software (WinDaq; DATAQ Instruments, Inc, Akron, Ohio). Grafts with flow less than 10 $\mathrm{mL} / \mathrm{min}$ and pulsatility index greater than 5 despite anastomotic revision were excluded from analysis $(n=2)$.

\section{Immunohistochemical Staining and Enzyme-Linked Immunosorbent Assay}

Surplus segments obtained from each bypass conduit just before the distal anastomosis were stored in Hanks balanced salt solution, embedded in cutting compound (Tissue-Tek O.C.T., Electron Microscopy Sciences, Hatfield, $\mathrm{Pa}$ ) and then frozen in liquid nitrogen. The percentages of the luminal circumference staining for the endothelial marker CD31 (R\&D Systems, Minneapolis, Minn) and tissue factor (United States Biological, Swampscott, Mass) were analyzed as described previously ${ }^{10}$ (Figure 1).

\section{Assays for Coagulation}

Tests of coagulation (international normalized ratio, partial thromboplastin time, levels of fibrinogen and the peptide fragment F1.2, and quantitative D-dimer levels) were obtained from citrated blood samples drawn just before skin incision (baseline), just after protamine administration, and on postoperative days 1,3 , and 30 . Platelet-poor plasma was obtained from a coronary sinus (CS) blood sample drawn by direct puncture with a 21-gauge needle 

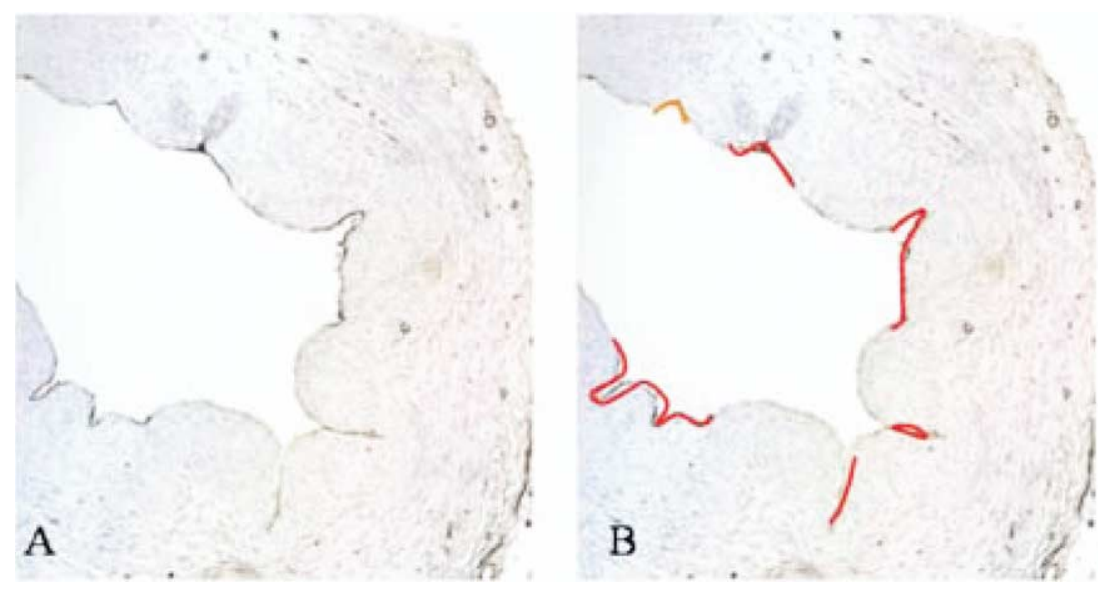

Figure 1. Surplus segments of each vein graft were procured intraoperatively and underwent immunohistochemical testing with CD31 monoclonal antibody. EC integrity was defined as percentage luminal circumference positive for CD31 according to image analysis software. A, As illustrated in this representative SVG section, CD31 staining was inconsistent on endoluminal surface of vein, suggesting endothelial disruption. In contrast, luminal staining was consistently positive in adventitial vessels of vasa vasorum, which were less exposed to procurement trauma. B, With image analysis, percentage endoluminal surface positive for CD31 was calculated. Original magnification $\times 10$.

approximately 30 minutes after CABG in a subset of subjects $(\mathrm{n}=$ 40). F1.2 levels were obtained with an enzyme-linked immunosorbent assay kit (Enzygnost F1.2 Micro; Dade Behring Inc, Deerfield, Ill) and reported as nanomoles per liter per milligram of protein. Comparison of CS blood with a simultaneously obtained aortic blood sample allowed the calculation of percentage transcardiac change as follows: (F1.2 in CS - F1.2 in aorta)/F1.2 in CS $\times 100 \%$.

\section{Assays for Aspirin Resistance}

The diagnosis of aspirin resistance was based on positive findings consistent with this diagnosis on at least two of three of the following assays.

Thrombelastography (TEG). We used a recently described modification of the TEG assay (Haemoscope Corporation, Niles, Ill) that allows clot formation to occur without thrombin generation in heparinized whole blood. ${ }^{14}$ The resulting clot maximum amplitude in this assay with added arachidonic acid $(0.5 \mu \mathrm{mol} / \mathrm{L}$, $\left.\mathrm{MA}_{\mathrm{AA}}\right)$ or without a platelet agonist $\left(\mathrm{MA}_{0}\right)$ was compared with the standard kaolin-activated (thrombin induced) TEG assay $\left(\mathrm{MA}_{\mathrm{KH}}\right)$. Aspirin resistance was defined by a value greater than $50 \%$ derived from a normalized formula: $\% \mathrm{MA}_{\mathrm{AA}}=\left(\left[\mathrm{MA}_{\mathrm{AA}}-\mathrm{M}_{\mathrm{A} 0}\right] /\right.$ $\left.\left[\mathrm{MA}_{\mathrm{KH}}-\mathrm{M}_{\mathrm{AO}}\right]\right) \cdot 100 \% .^{14}$

Whole-blood aggregometry (WBA). Impedance changes were assessed by WBA (Chronolog, Hawerton, Pa) at 6 minutes after the addition of $1-\mu \mathrm{g} / \mathrm{mL}$ and $5-\mu \mathrm{g} / \mathrm{mL}$ collagen. Aspirin resistance was defined as previously reported by our laboratory ${ }^{13}$ and others. ${ }^{15}$

11-Dehydro-thromboxane $B_{2}$ levels. Platelet poor serum was assayed for 11-dehydro-thromboxane $\mathrm{B}_{2}$ levels with an enzymelinked immunosorbent assay kit (Assay Designs Inc, Ann Arbor, Mich). Aspirin resistance was defined as an increase in serum levels greater than $25 \%$ relative to baseline. ${ }^{16}$

Whole-blood flow cytometry. In a subset of patients with and without aspirin resistance, blood was incubated in the presence and absence of arachidonic acid $(1.0 \mathrm{mmol} / \mathrm{L})$ for 2 minutes. Saturating concentrations of fluorescently labeled antibodies that bind to receptors expressed on platelets constitutively (CD41a; BD PharMingen, San Diego, Calif) or after activation of P-selectin (CD62P; BD PharMingen) were then added. After 20 minutes of incubation, the samples were fixed with $1 \%$ paraformaldehyde and stored at $4{ }^{\circ} \mathrm{C}$ to $8^{\circ} \mathrm{C}$ until analysis within 72 hours with a fluorescence-activated cell sorter (Becton-Dickinson FACScan; BD Immunocytometry Systems, San Jose, Calif). Aspirin resistance was defined as a $25 \%$ increase in expression of the CD62P receptor after stimulation ${ }^{13}$ (Figure 2).

\section{Postoperative Graft Follow-up}

SVG patency was determined by blinded review (C.W.) of an angiographic study acquired on a 16-slice multidetector CT scan. The protocol for these scans and methods for determining patency were as previously reported by our group. ${ }^{10,13,17}$

\section{Statistics}

The primary end point of this study was the risk of early graft failure in patients with both aspirin resistance and poor EC integrity in the SVG relative to patients with neither of these risks. Poor EC integrity was defined by CD31 staining less than $25 \%$ luminal circumference, the lowest quartile of our study population seen in preliminary studies. ${ }^{8}$ Given that aspirin resistance occurs in as many as $50 \%$ of patients after $\mathrm{CABG},{ }^{12}$ there was thus a $12.5 \%$ risk of both aspirin resistance and poor EC integrity occurring together, assuming these conditions to be independent. Assuming a strong relationship between these risks and SVG failure, a population of 210 patients was estimated to yield the minimum number of high-risk patients needed to demonstrate a $35 \%$ difference in the rate of early graft failure at $80 \%$ power and $P=.05$ (UCLA Department of Statistics. Power Calculator [http://www. stat.ucla.edu/]. Los Angeles, CA: UCLA Department of Statistics [updated 2004 August 18]. Available from: http://calculators.stat. ucla.edu/powercalc/).

Two-way analysis of variance was used to demonstrate a synergistic influence between risk factors on early graft failure. ${ }^{18}$ All candidate risk variables were subjected to univariate analysis by means of the Student $t$ test and the Fisher exact test for continuous and categorical variables, respectively. Multivariate analysis was then performed to assess for interactions not detected on the univariate analysis. Without dropping the significant variables 


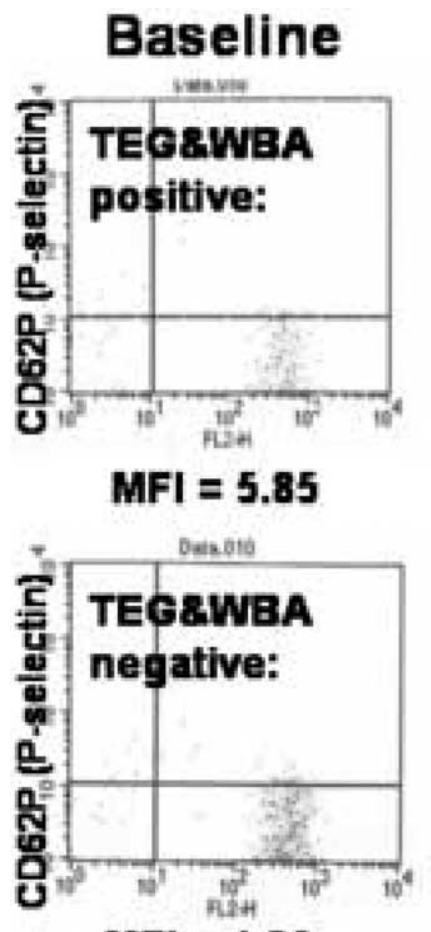

MFI $=\mathbf{4 . 2 6}$
AA $1.0 \mathrm{mM}$

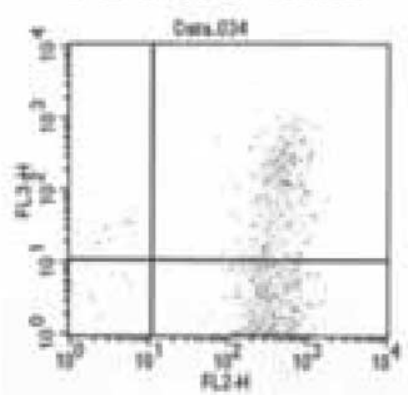

MFI $=149.30$

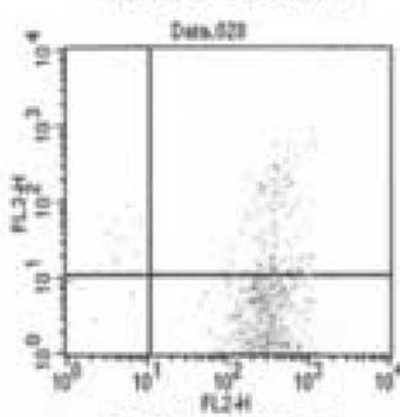

MFI $=\mathbf{1 6 . 8 5}$

Figure 2. Whole-blood flow cytometry was used to confirm diagnosis of aspirin resistance by monitoring platelets (anti-CD41a, $x$-axis) for change in P-selectin expression (anti-CD62P, y-axis) in response to arachidonic acid $(A A, 1.0 \mathrm{mmol} / \mathrm{L})$. These representative examples illustrate $\mathbf{3 0}$-fold increase in mean fluorescence intensity (MFI) for P-selectin when both TEG and WBA were positive for aspirin resistance after arachidonic acid stimulation relative to baseline. In contrast, when both functional assays showed aspirin responsiveness, there was little change in P-selectin after arachidonic acid.

obtained from univariate analysis, a stepwise multivariate analysis was performed by inserting all variables listed in Table 1 into the model and accepting variables for the next step that had $P$ values less than .99 , then .98 , and then .95 . A fourth analysis was performed with a $P=.05$ criterion for final retention of variables. The predictability of the model was determined by the c-statistic. Statistical analysis was performed using the InStat (GraphPad Software, Inc, San Diego, Calif) and MedCalc (MedCalc Software, Mariakerke, Belgium) statistical packages with the assistance of a biostatistician (L.N.).

The sponsors of this study had no role in the analysis or publication of these data.

\section{Results}

\section{Study Population}

CT angiographic follow-up was complete for 225 of 277 enrolled subjects (82\%). Patency of arterial grafts $(n=261$, 223 left internal thoracic artery, 22 right internal thoracic

artery, 16 radial artery) was not included in this analysis but was $100 \%$. Of 410 SVGs, 2 with intraoperative graft blood flow less than $10 \mathrm{~mL} / \mathrm{min}$ despite revision were excluded from analysis. Of the 408 SVGs analyzed, 16 grafts in 16 patients were found to be thrombosed: 6 of 184 grafts to the right coronary artery, 6 of 139 grafts to the circumflex, and 4 of 85 placed onto a diagonal coronary artery.

No differences with regards to demographic characteristics, preoperative risk factors, or medication use were noted between the 16 patients who had SVG thrombosis and those whose grafts remained patent. Intraoperative data such as ejection fraction, conduit diameter, target quality, and inotropic requirements were also similar (Table 1).

\section{Abnormalities in the Conduit}

SVG that failed had lower intraoperative blood flow than had those remaining patent, but the differences did not reach statistical significance $(37 \pm 29 \mathrm{~mL} / \mathrm{min}$ vs $47 \pm 32 \mathrm{~mL} /$ min). Pulsatility index and percentage diastolic flow, previously described proxies for grafts at risk for early closure, ${ }^{19-21}$ also failed to discriminate between grafts that thrombosed and those that remained patent (pulsatility index $2.4 \pm 1.4$ vs 2.2 \pm 0.9 , difference not significant, and percentage diastolic flow $51 \% \pm 19 \%$ vs $57 \% \pm 10 \%$, difference not significant).

The percentage EC integrity, determined in 380 total SVGs (191 patients), was 11\% (95\% confidence interval [CI] $-0.41 \%$ to $21.9 \%$ ) for the $16 \mathrm{SVGs}$ that failed, versus $51 \%$ (95\% CI $44.2 \%-56.0 \%$ ) for SVGs remaining patent $(P<.01$; Figure 1$)$, with no difference between open $(49 \% \pm$ $23 \%)$ and endoscopic $(51 \% \pm 30 \%)$ approaches. Tissue factor expression correlated with the EC disruption $(R=$ $0.65, P<.01$ ). Vein EC integrity less than $8 \%$ (first quartile) coincided with a graft failure rate of $28 \%$ and with a subendothelial pattern of expression (Figure 3).

\section{Abnormalities in Aspirin Responsiveness}

Aspirin resistance was diagnosed in 10 patients at baseline (4\%), in 22 patients (10\%) on day 1, and in 67 patients (30\%) on day 3 ; it had resolved in all but 7 patients $(2 \%)$ by day 30. A strong correlation was seen between the platelet response on day 3 to arachidonic acid by TEG and low-dose collagen by WBA (percentage change in $\mathrm{MA}_{\mathrm{AA}}$ and percentage impedance change low and high, respectively, $R=$ $0.72, P<.001)$. Patients demonstrating aspirin resistance according to both TEG and WBA also showed a significant rise in perioperative serum thromboxane $B_{2}$ levels relative to those with normal aspirin response (change from baseline $42 \% \pm 94 \%$ vs $-51 \% \pm 20 \%, P<.001)$. An analysis of platelet $\mathrm{P}$-selectin expression in response to arachidonic acid on flow cytometric analysis of an unselected subset of 20 study patients provided further confirmation of the diagnosis of aspirin resistance. An increase in P-selectin expres- 
TABLE 1. Preoperative demographic characteristics, intraoperative findings, and postoperative course

\begin{tabular}{|c|c|c|c|}
\hline & Status of $\mathbf{S}$ & ngiography & \\
\hline & Thrombosis $(\mathrm{n}=16)$ & All patent $(n=209)$ & $P$ value \\
\hline Age $(y$, mean $\pm S D)$ & $67 \pm 9$ & $69 \pm 12$ & NS \\
\hline Sex $(\%$ male $)$ & 61 & 66 & NS \\
\hline Active smoker (\%) & 15 & 25 & NS \\
\hline Hypertension (\%) & 90 & 84 & NS \\
\hline Previous stroke (\%) & 16 & 10 & NS \\
\hline Diabetes (\%) & 45 & 42 & NS \\
\hline Ejection fraction $<40 \%{ }^{*}(\%)$ & 20 & 18 & NS \\
\hline Preoperative medications & & & \\
\hline$\beta$-Blockers (\%) & 91 & 87 & NS \\
\hline Aspirin (\%) & 90 & 94 & NS \\
\hline Angiotensin-converting enzyme inhibitors (\%) & 60 & 53 & NS \\
\hline Statins $(\%)$ & 75 & 78 & NS \\
\hline Intraoperative factors & & & \\
\hline Target size $<1.5 \mathrm{~mm}(\%)$ & 56 & 32 & .03 \\
\hline Poor target quality (\%) & 25 & 29 & NS \\
\hline SVG/coronary diameter $(\%$, mean $\pm S D)$ & $3.78 \% \pm 0.79 \%$ & $2.63 \% \pm 1.01 \%$ & $<.05$ \\
\hline Circumflex target $(\%)$ & 38 & 33 & NS \\
\hline Wall motion abnormality absent in target region (\%)† & 49 & 32 & .08 \\
\hline Graft flow $(\mathrm{mL} / \mathrm{min}$, mean $\pm \mathrm{SD})$ & $37 \pm 29$ & $47 \pm 32$ & NS \\
\hline SVG integrity (\%CD31) & 11 & 51 & .01 \\
\hline Required intra-aortic balloon pump (\%) & 18 & 16 & NS \\
\hline Postoperative factors & & & \\
\hline Blood loss in $24 \mathrm{~h}(\mathrm{~mL}$, mean $\pm \mathrm{SD})$ & $753 \pm 146$ & $810 \pm 114$ & NS \\
\hline Given blood products $(\%)$ & 49 & 57 & NS \\
\hline Fibrinogen (mg/dL) $\ddagger$ & $680 \pm 102$ & $705 \pm 115$ & NS \\
\hline Prothrombin time $(s) \ddagger$ & $12.8 \pm 0.9$ & $13.4 \pm 0.8$ & NS \\
\hline $\mathrm{D}$-Dimer $(\mathrm{ng} / \mathrm{mL}$, mean $\pm \mathrm{SD}) \ddagger$ & $1954 \pm 217$ & $1687 \pm 255$ & NS \\
\hline Aspirin resistance at any point (\%) & 45 & 20 & $<.05$ \\
\hline Aspirin resistance on day $1(\%)$ & 25 & 7.6 & .02 \\
\hline Length of intubation ( $h$, mean \pm SD) & $17 \pm 12$ & $16 \pm 14$ & NS \\
\hline Postoperative low cardiac index§ (\%) & 9 & 8 & NS \\
\hline Postoperative stay (d, mean \pm SD) & $6.1 \pm 1.1$ & $6.2 \pm 1.9$ & NS \\
\hline
\end{tabular}

NS, Not significant. *Ejection fraction determined by intraoperative transesophageal echocardiography. $\dagger$ Intraoperative echocardiographic examination showing hypokinesis or akinesis of wall motion in myocardial region served by target coronary artery. $\$$ Peak (most hypercoagulable) values for entire postoperative period. §Cardiac index less than $2.0 \mathrm{~L} /\left(\mathrm{min} \cdot \mathrm{m}^{2}\right)$.

sion in response to AA greater than 10-fold was seen in 4 of 6 patients with a diagnosis of aspirin resistance according to the TEG and WBA (Figure 2). On the other hand, only 1 of the 14 patients thought to be aspirin sensitive showed a similar change in $\mathrm{P}$-selectin expression $(P<.02$, Fisher exact test). The incidence of aspirin resistance was $45 \%$ among those found to have early graft failure, versus $20 \%$ in those in whom all grafts were patent $(P<.05$, Fisher exact test).

\section{Abnormalities in Coagulation}

Routine assays of the coagulation cascade (prothrombin time, partial thromboplastin time, fibrinogen level, D-dimers) failed to discriminate patients with eventual SVG failure (data not shown). In contrast, transcardiac thrombin formation (as- sessed by F1.2 levels in the CS compared with the aorta) was significantly elevated in those patients who had SVG failure relative to those with all patent grafts (percentage arteriovenous difference $112 \% \pm 34 \%, \mathrm{n}=4$, vs $19 \% \pm$ $41 \%, \mathrm{n}=36, P<.05)$. Despite equivalent mean intraoperative heparin levels $(2.0 \pm 0.6 \mu \mathrm{g} / \mathrm{mL}$ vs $1.9 \pm 0.4 \mu \mathrm{g} / \mathrm{mL})$, transcardiac change in F1.2 was significantly increased in patients grafted with veins in the lowest quartile of EC integrity relative to the upper three quartiles (increase in CS vs aorta $122.4 \% \pm 95.9 \%$ vs $25.4 \% \pm 32.7 \%, P<.05)$.

\section{Influence of Combined Abnormalities on Graft Thrombosis}

A total of 32 SVGs in 28 patients met the study criteria for highest risk, EC integrity in the lowest quartile in the setting 

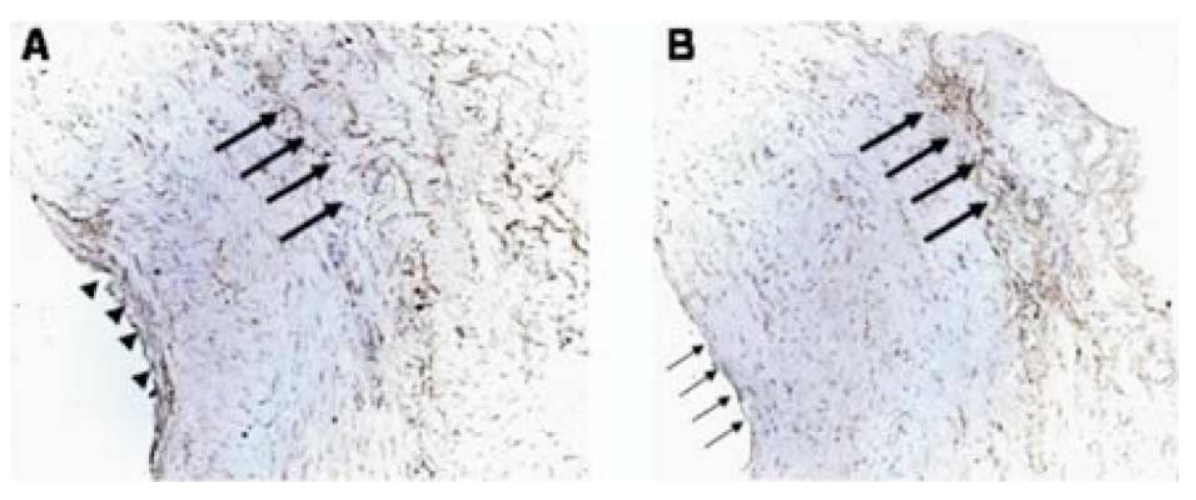

Figure 3. Tissue factor was constitutively expressed in adventitia (thick arrows) of surplus vein segments taken from each bypass graft analyzed by immunohistochemistry. Subendothelial pattern of tissue factor expression (A, arrowheads) was detected in 52 vein segments with more severe disruption of EC integrity $(12 \%, 95 \% \mathrm{Cl}-2 \%$ to $26 \%$ ). By comparison, vein segments that did not show this pattern of tissue factor expression ( $B$, thin arrows) showed better EC integrity $(49.6 \%, 95 \%$ Cl $34.4 \%$ $60.9 \%, n=355$ ).

of aspirin resistance; early attrition was discovered before hospital discharge in 10 of these patients (37\%; Figure 4). Graft failure in patients with both these risk factors comprised a majority $(63 \%)$ of the cases of graft failure, indicating a positive predictive value of $63 \%$ (95\% CI $35 \%$ $85 \%$ ). On the other hand, 25 patients had neither of these two risk factors and all patent grafts, indicating a negative predictive value of $91 \%$ (95\% CI 87\%-95\%) and a significant improvement in patency relative to the high risk group $(P<.001$, Fisher exact test $)$. EC disruption and aspirin

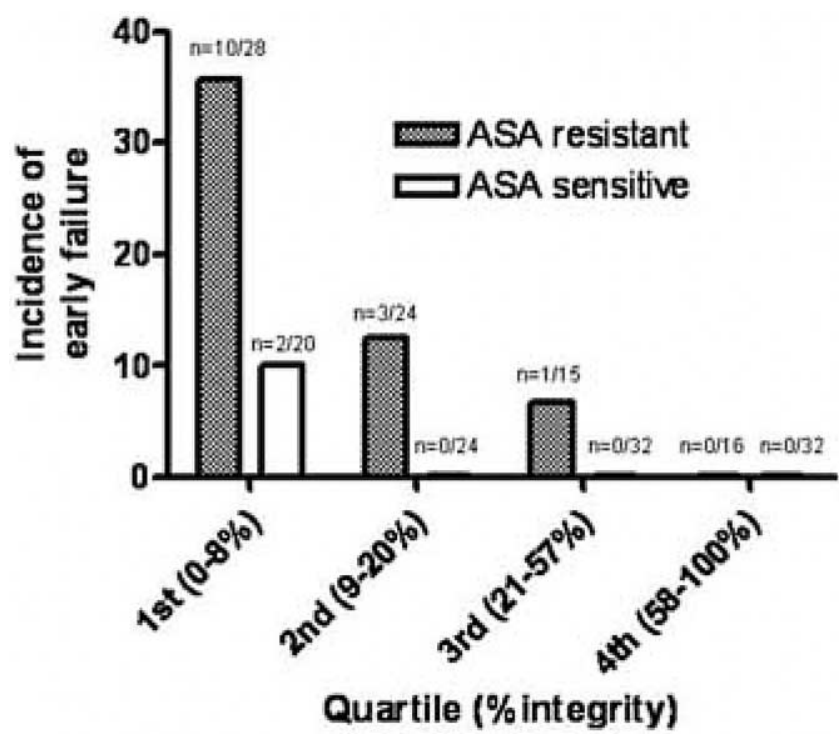

Figure 4. Although aspirin (ASA) monotherapy was sole antithrombotic strategy used during this study, postoperative aspirin resistance was significantly associated with failure only in vein grafts in lowest quartile of EC integrity (<9\%). Additionally, very few grafts in this lowest quartile failed if aspirin sensitivity was preserved after OPCAB. Presence of both of these risk factors dramatically increased the risk of graft failure. Synergistic interaction of ASA-R and EC disruption was confirmed by paired analysis of variance test. resistance had a synergistic effect on graft failure ( $\mathrm{F}$ score 15.91, $P<.01$, 2-way analysis of variance). Six variables (listed in Table 1) were found to be different between groups on univariate analysis. However, only three variables were retained as independent predictors after multivariate, logistic regression analysis: EC disruption in the SVG, aspirin resistance on day 1, and target diameter, with odds ratios of 5.98 (95\% CI 2.15-10.46, $P<.002$ ), 2.59 (95\% CI 1.13-5.95, $P<.025)$, and 0.035 (95\% CI $0.002-$ $0.734, P<.03)$, respectively. The presence of aspirin resistance at any perioperative time point was excluded because of a close correlation with other independent predictors and a model fitting strategy that discards repeated information. The $c$ index for the final model was 0.931 .

\section{Discussion}

The major finding of this study was that insufficient suppression of coagulation from aspirin (aspirin resistance) developing early after OPCAB and EC disruption in the SVG synergistically increased the risk of early graft thrombosis. In the 19th century, Virchow proposed that abnormalities in coagulation (eg, aspirin resistance) and EC integrity are critical factors for vascular thrombosis, yet no previous study has established a direct link between this risk combination and early SVG failure. Although graft failure in the first postoperative week is frequently blamed on surgical technique, we minimized this issue as a confounding variable by the routine use of intraoperative flow measurements. This protocol led to the revision or exclusion of defective grafts at a rate consistent with previous reports. ${ }^{19-21}$ Within this context, we did not find an increased rate of early failure among grafts that were more technically demanding during OPCAB, such as internal thoracic artery or grafts to the circumflex artery, suggesting that we were able to focus our analysis on risk factors related to the conduit or patient.

Despite a well-described tendency toward EC disruption $^{9,10}$ and a higher rate of failure than with the internal 
thoracic artery, ${ }^{5-7}$ SVG use remains prevalent because of familiarity, convenience, and acceptable intermediate-term results. EC injury may be caused by high-pressure saline solution infusion, abrasion by passage of instruments through the lumen, or by extraluminal crush injury (eg, metallic clamps or forceps) during procurement. Immediately after grafting, platelets adhere to collagen in the SVG exposed by EC disruption. ${ }^{22}$ Injured veins rapidly express tissue factor, ${ }^{23}$ triggering local thrombin formation. In vitro models show that the simultaneous exposure of platelets to collagen and thrombin within the damaged SVG is likely to activate platelets irregardless of aspirin use, ${ }^{24}$ setting up a positive feedback loop for further thrombin formation. ${ }^{22}$ The peptide fragment F1.2 is cleaved from prothrombin during the generation of thrombin and is a proven risk marker for graft thrombosis. ${ }^{25}$ Determining the CS level of this marker may provide a means of quantifying platelet and thrombin activation within the upstream SVG that occurs after the reversal of heparin. Although ischemia and native coronary endothelial disease have also been reported to affect thrombin formation, the disrupted vein graft probably has a disproportionate impact. Acute vascular injury has been shown to be a major stimulus for regional thrombin generation in animal models ${ }^{26}$ and in patients undergoing intracoronary stenting. ${ }^{27}$

Early SVG failure most often occurs without detectable signs or symptoms, which limits understanding about its exact timing. For practical reasons, we measured the CS F1.2 level 30 minutes after heparin reversal. The notion that this type of intraoperative assessment is able to reliably discriminate the risk of SVG thrombosis remains speculative. However, evidence suggests that the risk of thrombosis is maximal at initial graft perfusion. First, platelet inhibition improves graft patency only when given early postoperatively. Randomized trials have shown that aspirin initiated 48 hours or later after CABG does not influence the frequency of new SVG occlusions. ${ }^{11}$ Second, EC healing of the SVG also occurs within 2 to 3 days,${ }^{28}$ suggesting that the impact of SVG injury during procurement subsides within this same early timeframe. The ability of CS F1.2 levels immediately after OPCAB to serve as an integrated predictor of early SVG failure requires confirmation in future multivariate analysis from our ongoing study.

The importance of EC disruption and aspirin resistance suggests that less traumatic vein harvesting and alternative antithrombotic strategies might help to improve patency. Avoidance of pressure injury during preparation of the SVG could reduce the expression of tissue factor ${ }^{23}$ and thus thrombin formation after perfusion. Heparin minimizes thrombin formation during cardiopulmonary bypass ${ }^{29}$ but did not do so within the SVG during this OPCAB study. An agent that is able to inhibit clot-bound thrombin, such as bivalirudin, may provide more effective intraoperative anticoagulation for the SVG graft. Bivalirudin improved SVG patency in a randomized comparison with heparin during OPCAB. ${ }^{30}$ Perhaps by inhibition of the thrombin receptor on the platelet, intraoperative aprotinin use may provide another adjunct to promote SVG patency. ${ }^{31}$ Finally, clopidogrel more effectively inhibits platelets exposed to both collagen and thrombin in vitro than does aspirin. ${ }^{22}$ However, clopidogrel increases the risk of bleeding and potentially of death after cardiac surgery. ${ }^{32}$ Targeting clopidogrel to only those with increased transcardiac thrombin formation might provide a rational basis for accepting that risk.

The lack of clinical validation of our methods is the main limitation of this study. First, the best diagnostic test for aspirin resistance remains uncertain. We addressed this problem by considering the diagnosis of aspirin resistance to be established only if findings were compatible in two of three separate assays performed in all patients. The strong correlation between the complementary methods used to diagnose aspirin resistance and flow cytometry, a more established method of quantifying platelet function, justifies confidence regarding our diagnostic criteria. Second, because the risk for early SVG failure is not limited to the period before hospital discharge, most previous studies investigating early graft patency have obtained angiography at 1 to 3 months. Our protocol of predischarge CT tomographic angiography provided a higher and more uniform follow-up, which we considered an acceptable tradeoff. Finally, determining EC integrity on the basis of biopsy samples from the distal portion of each graft may not accurately reflect heterogeneous damage to other portions of the vein. Differential handling of the actual conduit used for bypass than of the discarded, surplus segments that were analyzed might augment the tendency of our report to overestimate the severity of EC injury. Future emphasis in this ongoing study will be placed on real-time methods of assessing EC integrity in the whole graft and on longer term follow-up of patency. Although mechanistically consistent, our data on regional thrombin formation as a cause of graft failure should be considered only as hypothesis generating pending prospective confirmation with these methodologic improvements.

In conclusion, in this study the combination of acquired aspirin resistance and EC disruption was strongly associated with intraluminal thrombin generation and the risk of early vein graft thrombosis. Exposed collagen and tissue factor within the damaged vein may create a regional burst in thrombin formation that contributes to the development of aspirin resistance. We studied only patients undergoing OPCAB order to eliminate potential confounding variables associated with cardiopulmonary bypass. However, these data suggest that aggressive prevention, directed by screening for conduit EC damage and transcardiac coag- 
ulation pathway activation, may provide the best method for improving vein graft patency after $\mathrm{CABG}$, on pump or off. ${ }^{2}$

\section{References}

1. Yun KL, Wu Y, Aharonian V, Mansukhani P, Pfeffer TA, Sintek CF, et al. Randomized trial of endoscopic versus open vein harvest for coronary artery bypass grafting: six-month patency rates. $J$ Thorac Cardiovasc Surg. 2005;129:496-503.

2. Turitto VT, Hall CL. Mechanical factors affecting hemostasis and thrombosis. Thromb Res. 1998;92(6 Suppl 2)S25-31.

3. Jackson ZS, Ishibashi H, Gotlieb AI, Langille BL. Effects of anastomotic angle on vascular tissue responses at end-to-side arterial grafts. $J$ Vasc Surg. 2001;34(2):300-7.

4. Peterson E, Mark DB. Off-pump bypass surgery-ready for the big dance? JAMA. 2004;291:1897-9.

5. Khan NE, De Souza A, Mister R, Flather M, Clague J, Davies S, et al. A randomized comparison of off-pump and on-pump multivessel coronary-artery bypass surgery. N Engl J Med. 2004;350:21-8.

6. Puskas JD, Williams WH, Mahoney EM, Huber PR, Block PC, Duke PG, et al. Off-pump vs conventional coronary artery bypass grafting: early and 1-year graft patency, cost, and quality-of-life outcomes: a randomized trial. JAMA. 2004;291:1841-9.

7. Widimsky P, Straka Z, Stros P, Jirasek K, Dvorak J, Votava J, et al. One-year coronary bypass graft patency: a randomized comparison between off-pump and on-pump surgery angiographic results of the PRAGUE-4 trial. Circulation. 2004;110:3418-23.

8. Poston R, White C, Read K, Gu J, Lee A, Avari T, et al. Virchow triad, but not use of an aortic connector device, predicts early graft failure after off-pump coronary bypass. Heart Surg Forum. 2004;7:123-8.

9. Thatte HS, Khuri SF. The coronary artery bypass conduit: I. Intraoperative endothelial injury and its implication on graft patency. Ann Thorac Surg. 2001;72:S2245-52; discussion S2267-70.

10. Manchio JV, Gu J, Romar L, Brown J, Gammie J, Pierson RN, 3rd et al. Disruption of graft endothelium correlates with early failure after off-pump coronary artery bypass surgery. Ann Thorac Surg. 2005;79: 1991-8.

11. Stein PD, Dalen JE, Goldman S, Schwartz L, Theroux P, Turpie AG. Antithrombotic therapy in patients with saphenous vein and internal mammary artery bypass grafts. Chest. 1995;108 4 Suppl:424S-30S.

12. Zimmermann N, Wenk A, Kim U, Kienzle P, Weber AA, Gams E, et al. Functional and biochemical evaluation of platelet aspirin resistance after coronary artery bypass surgery. Circulation. 2003;108:542-7.

13. Poston R, Gu J, Manchio J, Lee A, Brown J, Gammie J, et al. Platelet function tests predict bleeding and thrombotic events after off-pump coronary bypass grafting. Eur J Cardiothorac Surg. 2005;27:584-91.

14. Craft RM, Chavez JJ, Bresee SJ, Wortham DC, Cohen E, Carroll RC. A novel modification of the Thrombelastograph assay, isolating platelet function, correlates with optical platelet aggregation. J Lab Clin Med. 2004;143:301-9.

15. Friend M, Vucenik I, Miller M. Research Pointers: Platelet responsiveness to aspirin in patients with hyperlipidaemia. BMJ. 2003;326: 82-3.

16. Eikelboom JW, Hirsh J, Weitz JI, Johnston M, Yi Q, Yusuf S. Aspirin-resistant thromboxane biosynthesis and the risk of myocardial infarction, stroke, or cardiovascular death in patients at high risk for cardiovascular events. Circulation. 2002;105:1650-5.

17. Frazier AA, Qureshi F, Read KM, Gilkeson RC, Poston RS, White CS. Coronary artery bypass grafts: assessment with multidetector CT in the early and late postoperative settings. Radiographics. 2005;25:881-96.

18. Slinker BK. The statistics of synergism. J Mol Cell Cardiol. 1998;30: 723-31.

19. Morota T, Duhaylongsod FG, Burfeind WR, Huang CT. Intraoperative evaluation of coronary anastomosis by transit time ultrasound flow measurement. Ann Thorac Surg. 2002;73:1446-50.

20. Leong DK, Ashok V, Nishkantha A, Shan YH, Sim EK. Transit-time flow measurement is essential in coronary artery bypass grafting. Ann Thorac Surg. 2005;79:854-7.
21. D'Ancona G, Karamanoukian HL, Ricci M, Schmid S, Bergsland J, Salerno TA. Graft revision after transit time flow measurement in off-pump coronary artery bypass grafting. Eur J Cardiothorac Surg. 2000;17:287-93

22. Vanschoonbeek K, Feijge MA, Van Kampen RJ, Kenis H, Hemker $\mathrm{HC}$, Giesen PL, et al. Initiating and potentiating role of platelets in tissue factor-induced thrombin generation in the presence of plasma: subject-dependent variation in thrombogram characteristics. $J$ Thromb Haemost. 2004;23:476-84.

23. Muluk SC, Vorp DA, Severyn DA, Gleixner S, Johnson PC, Webster MW. Enhancement of tissue factor expression by vein segments exposed to coronary arterial hemodynamics. J Vasc Surg. 1998;27:521-7.

24. Alberio L, Safa O, Clemetson KJ, Esmon CT, Dale GL. Surface expression and functional characterization of alpha-granule factor $\mathrm{V}$ in human platelets: effects of ionophore A23187, thrombin, collagen, and convulxin. Blood. 2000;95:1694-702.

25. Moor E, Hamsten A, Blomback M, Herzfeld I, Wiman B, Ryden L. Haemostatic factors and inhibitors and coronary artery bypass grafting: preoperative alterations and relations to graft occlusion. Thromb Haemost. 1994;72:335-42.

26. Hatton MW, Moar SL, Richardson M. Deendothelialization in vivo initiates a thrombogenic reaction at the rabbit aorta surface. Correlation of uptake of fibrinogen and antithrombin III with thrombin generation by the exposed subendothelium. Am J Pathol. 1989;135:499508.

27. Mizuno O, Hojo Y, Ikeda U, Katsuki T, Fukazawa H, Kurosaki K, et al. Assessment of coagulation and platelet activation in coronary sinus blood induced by transcatheter coronary intervention for narrowing of the left anterior descending coronary artery. Am J Cardiol. 2000;85:154-60.

28. Ehsan A, Mann MJ, Dell'Acqua G, Tamura K, Braun-Dullaeus R, Dzau VJ. Endothelial healing in vein grafts: proliferative burst unimpaired by genetic therapy of neointimal disease. Circulation. 2002; 105:1686-92.

29. Koster A, Fischer T, Praus M, Haberzettl H, Kuebler WM, Hetzer R, et al. Hemostatic activation and inflammatory response during cardiopulmonary bypass: impact of heparin management. Anesthesiology. 2002;97:837-41

30. Merry AF, Raudkivi PJ, Middleton NG, McDougall JM, Nand P, Mills $\mathrm{BP}$, et al. Bivalirudin versus heparin and protamine in off-pump coronary artery bypass surgery. Ann Thorac Surg. 2004;77:925-31.

31. Poston R, White C, Gu J, Brown J, Gammie J, Pierson R, et al. Hemostatic and antithrombotic effects of aprotinin during off-pump coronary artery bypass grafting: results from a randomized prospective trial. Ann Thorac Surg. In press 2005.

32. Ascione R, Ghosh A, Rogers CA, Cohen A, Monk C, Angelini GD. In-hospital patients exposed to clopidogrel before coronary artery bypass graft surgery: a word of caution. Ann Thorac Surg. 2005;79:1210-6.

\section{Discussion}

Dr Steven Bolling (Ann Arbor, Mich). Poston and coauthors should be congratulated. They have demonstrated satisfactory evidence to support their conclusion that the combination of aspirin resistance and endothelial disruption lead to early graft failure. I have a number of questions.

Dr Poston, you used a somewhat complicated method to determine aspirin resistance involving TEG, WBA, and then flow. As you know, it is somewhat controversial to determine aspirin responsiveness. Did you not consider a more sensitive assay, such as platelet function analyzer (PFA-100), which can show aspirin resistance and aspirin semiresponsivenesss?

Second, did you think that in many of these patients there may be genetic polymorphism, especially at the $\mathrm{P} 1 \mathrm{~A} 2$ receptor, that can be measured in these patients to determine aspirin responsiveness?

Third, have you changed your clinical practice because of this study? Are we to measure F1.2 in terms of thrombin generation? 
Have you changed your technique of vein harvest? What is your clinical use of aspirin for these patients?

Dr Robert S. Poston, Jr (Baltimore, Md). With regard to the first question about the best diagnostic method for aspirin resistance, clearly you have hit on a valid issue. The best method to diagnose aspirin resistance is not clear. We chose our assays on the basis of them being conducted at point of care, and the PFA-100 that you mentioned is a promising test that also is at point of care. I think that is critical if we want to have these assays be clinically relevant and translate into clinical care.

Probably the best, most accurate method of diagnosing aspirin resistance is flow cytometry, but that is never going to be a clinically validated assay because it takes a 2- to 3-day turnaround, at best, to do such assays. So a point-of-care test that can yield results in 20 or 30 minutes is, I think, mandatory.

The PFA-100 is nice because it has a shear effect that induces platelet reactivity and is probably more relevant for the surgical patient. We didn't use it in our particular series of studies here because we were fairly convinced with our diagnostic method. We used, as we said, two point-of-care tests that correlated well with each other. They correlated with a clinical event that happens fairly frequently, graft thrombosis, and also correlated with the more sensitive measures we had, flow cytometry and thromboxane formation. So we felt good about our method of diagnosis. Certainly there are other assays that may be as good.

The second issue is the genetics. I think that is a critical point as well. If you want to devise a strategy to improve outcome, it is probably best to know beforehand whether aspirin resistance is going to develop, according to genetics or an understanding of the mechanism of aspirin resistance, and stop it before it occurs rather than to try to change your management after detecting it in a postoperative period. So that is the plan of our group, and we have those studies ongoing.

With regard to changing clinical practice, we are not approved by the institutional review board to use these results to change practice, but that is a plan in the future. I think that assessing F1.2 in the CS, because it seems to integrate all of the risk factors for graft failure, would be an important trial to do in the future, and with care adjusted according to the results of that assay.

Finally, with regard to aspirin use versus other antiplatelet agents, we desperately need agents other than aspirin because of this concern about the development of aspirin resistance after surgery. I don't think clopidogrel (Plavix) is the answer. The therapeutic window of clopidogrel is poor. You have to load it. Many surgeons just use a standard 75-mg dose.

A recent article in this Journal said that this doesn't affect platelet function, and we found that as well, that if you don't give a loading dose at 1 and 2 weeks out, you have no effect on aggregation in a surgical patient. It is different in a patient who hasn't undergone surgery, which is where our belief that 5 days with $75 \mathrm{mg}$ is all you need to get an effect. That is where that comes from.

There are companies that are working on an intravenous form of a clopidogrel analog that is short acting, and I think that has a lot of promise. Also, there may be strategies other than heparinization with bivalirudin that might have some more effect on thrombin generation in the graft as well.

Dr Frank Sellke (Boston, Mass). In follow-up to your last statement, is there any evidence that those patients who have or are suspected of having aspirin resistance have improved graft patency with the addition of clopidogrel? Looking at direct platelet function, there doesn't seem to be any difference, but has anybody looked at actual graft patency in these patients?

Dr Poston. The only thing that patients used in this trial was aspirin. So I can't answer that from my own data. Your point is that there are no data indicating that clopidogrel improves patency. I think that is a valid point, mainly because it hasn't been used the way it should be used, which is as a 300-mg or perhaps even 600 -mg load in the immediate postoperative period. So I think using it at $75 \mathrm{mg} /$ day, the way it is in common practice, is not likely to have an effect. I do think that it is going to be important to have a better antiplatelet therapy titrated according to some kind of platelet function analysis or coagulation analysis to optimize our results.

Dr Guo-Wei He (Hong Kong, China). Since arterial grafting does not reach $100 \%$ patency rates, do you think aspirin resistance is also related to the occlusion of the arterial graft such as a free graft of the radial artery as well?

Dr Poston. Well, we used arterial grafts in this study. We didn't report it. Every one of our arterial grafts was patent. So I can't relate aspirin resistance to arterial graft problems. I think that probably based on my finding that there wasn't any influence of aspirin resistance on arterial graft patency that probably when arterial grafts fail it is technical, more likely, than it would be with a vein, which has a lot more thrombogenicity than an artery, and so the role of aspirin resistance and graft thrombogenicity plays a higher role with the vein. 